\title{
Multi-configurational Ehrenfest simulations of ultrafast nonadiabatic dynamics in a charge-transfer complex
}

Cite as: J. Chem. Phys. 149, 244107 (2018); https://doi.org/10.1063/1.5062608

Submitted: 25 September 2018 . Accepted: 19 November 2018 . Published Online: 27 December 2018

Tianji Ma, Matteo Bonfanti (D), Pierre Eisenbrandt, Rocco Martinazzo (D), and Irene Burghardt (D)
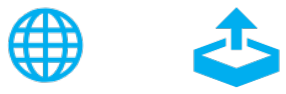

\section{ARTICLES YOU MAY BE INTERESTED IN}

An efficient approximate algorithm for nonadiabatic molecular dynamics

The Journal of Chemical Physics 149, 244117 (2018); https://doi.org/10.1063/1.5046757

On-the-fly ab initio semiclassical evaluation of time-resolved electronic spectra

The Journal of Chemical Physics 149, 244115 (2018); https://doi.org/10.1063/1.5054586

Analysis of trajectory similarity and configuration similarity in on-the-fly surface-hopping simulation on multi-channel nonadiabatic photoisomerization dynamics

The Journal of Chemical Physics 149, 244104 (2018); https://doi.org/10.1063/1.5048049

PHYSICS TODAY WHITEPAPERS
ADVANCED LIGHT CURE ADHESIVES

Take a closer look at what these

environmentally friendly adhesive systems can do
READ NOW

PRESENTED BY 8. MASTERROND 


\title{
Multi-configurational Ehrenfest simulations of ultrafast nonadiabatic dynamics in a charge-transfer complex
}

\author{
Tianji Ma, ${ }^{1}$ Matteo Bonfanti, ${ }^{1, a)}$ Pierre Eisenbrandt, ${ }^{1}$ Rocco Martinazzo, ${ }^{2}$ \\ and Irene Burghardt ${ }^{1, b)}$ \\ ${ }^{1}$ Institute of Physical and Theoretical Chemistry, Goethe University Frankfurt, Max-von-Laue-Str. 7, \\ D-60438 Frankfurt/Main, Germany \\ ${ }^{2}$ Department of Chemistry, Università degli Studi di Milano, Via Golgi 19, 20133 Milano, Italy
}

(Received 25 September 2018; accepted 19 November 2018; published online 27 December 2018)

\begin{abstract}
Multi-configurational Ehrenfest (MCE) approaches, which are intended to remedy the lack of correlations in the standard mean-field Ehrenfest method, have been proposed as coherent-state based ansätze for quantum propagation [D. V. Shalashilin, J. Chem. Phys. 130, 244101 (2009)] and also as the classical limit of the variational Gaussian-based multiconfiguration time dependent Hartree (G-MCTDH) method [S. Römer and I. Burghardt, Mol. Phys. 111, 3618 (2013)]. In the present paper, we establish the formal connection between these schemes and assess the performance of MCE for a coherent-state representation of the classical-limit subsystem. As a representative model system, we address the ultrafast, coherent charge transfer dynamics in an oligothiophene-fullerene donoracceptor complex described by a two-state linear vibronic coupling model. MCE calculations are compared with reference calculations performed with the MCTDH method, for 10-40 vibrational modes. Beyond a dimensionality of 10 modes, it is shown that the correct representation of electronic coherence depends crucially on the sampling of initially unoccupied Gaussians. Published by AIP Publishing. https://doi.org/10.1063/1.5062608
\end{abstract}

\section{INTRODUCTION}

Mixed quantum-classical dynamical approaches are highly useful to describe situations where a partitioning into a quantum subsystem and a classical environment is sought for, as exemplified by solute-solvent dynamics, adsorbatesurface processes, or the dynamics of electronic excitations in molecular materials. Various quantum-classical approaches have been developed, including the Ehrenfest approach, ${ }^{1-4}$ the surface-hopping (SH) approach, ${ }^{5-9}$ and the QuantumClassical Liouville Equation (QCLE). ${ }^{10-15}$ These methods differ in their capacity to describe correlations between the quantum and classical subsystems; notably, the QCLE provides the most detailed description of the quantum-classical boundary. By contrast, the Ehrenfest approach is based upon a mean-field approximation, combined with a classical path picture. This method is self-consistent in the same sense as the time-dependent self-consistent field (TDSCF), ${ }^{16-18}$ or the Time-Dependent Hartree (TDH) wavefunction approach, but exhibits various shortcomings for the same reasons. Notably, Ehrenfest dynamics has been shown to violate the principle of detailed balance, ${ }^{19,20}$ i.e., it turns out that the quantum subsystem evolves towards an artificial equilibrium state whose temperature is higher than the temperature of the classical environment. The SH approach, usually in the form of Tully's Fewest Switches Surface Hopping

a)Electronic mail: bonfanti@chemie.uni-frankfurt.de

b)Electronic mail: burghardt@chemie.uni-frankfurt.de
(FSSH), ${ }^{6,8}$ in turn complies with detailed balance, ${ }^{21}$ at least to a good approximation, ${ }^{22}$ but neglects the role of quantum coherence in the transition between states of the quantum subsystem. Augmented approaches like the Consensus Surface Hopping (CSH) scheme ${ }^{23}$ and related approaches ${ }^{24}$ improve on $\mathrm{SH}$ dynamics by rigorously connecting to the QCLE.

The present paper is concerned with a generalization of the Ehrenfest approach, based upon the observation that the mean-field Ehrenfest scheme is a single-configurational approach whose shortcomings could be remedied by multiconfigurational extensions. To this end, the wavefunction is represented as a superposition of Ehrenfest configurations, following work by Shalashilin. ${ }^{25,26}$ As in the context of more accurate multiconfigurational wavefunction methods, notably the Multi-Configuration Time-Dependent Hartree (MCTDH) method, ${ }^{27,28}$ the concept is to introduce correlations by creating superpositions of configurations. In Refs. 25 and 26, two types of such a multi-configuration Ehrenfest (MCE) approach have been proposed, exhibiting interacting Ehrenfest configurations (denoted MCE version 1, or MCEv1) ${ }^{25}$ or else independently evolving Ehrenfest configurations (denoted MCE version 2, or MCEv2). ${ }^{26}$ Furthermore, we have shown in Ref. 29 that MCE can be understood as the quantum-classical limit of the Gaussian-based Multi-Configuration Time-Dependent Hartree (G-MCTDH) method. ${ }^{30}$ The latter MCE variant represents a rigorous quantum-classical limit, whereas MCEv1 and MCEv2 refer to finite-width wavepackets and therefore represent quantum-semiclassical rather than quantum-classical approaches. 
Against this background, the objective of the present paper is twofold. First, we aim to connect the development of Ref. 29 with the MCEv1 and MCEv2 methods of Refs. 25 and 26. In practice, the rigorous quantum-classical limit representation of Ref. 29 is replaced by a Gaussian wavepacket (GWP) based representation, which at least, in principle, permits a full description of phase interference between Ehrenfest configurations. We will show that this leads to a formulation which is close to the MCEv2 approach. Second, we will assess the performance of MCE in a case study of nonadiabatic dynamics in a high-dimensional molecular system. Specifically, we refer to a donor-acceptor (DA) complex describing charge transfer between oligothiophene and fullerene moieties, which we studied previously in Ref. 31. In this system, superpositions of electronic configurations are shown to persist in time and the standard meanfield Ehrenfest approach is found to fail. In this context, we examine the role of configurational overlap and assess the use of phase space sampling techniques, along the lines of the compression methods employed in Refs. 25, 26, 32, and 33 .

While the present paper does not address an on-the-fly implementation, our results are relevant in the context of existing techniques, notably the $a b$ initio Multiple Cloning Multiconfigurational Ehrenfest (AIMC-MCE) approach ${ }^{32}$ as compared with $a b$ initio Multiple Spawning (AIMS) $)^{34-37}$ and the direct-dynamics variational Multiconfigurational Gaussian (DD-vMCG) approach. ${ }^{38-41}$ In on-the-fly calculations, comparatively sparse trajectory ensembles are employed such that the choice of the propagation method plays an important role.

The remainder of this article is organized as follows. Section II reviews the MCE approach from the viewpoint of our earlier development of Ref. 29 and shows how the latter formulation is augmented so as to correspond to finite-width GWPs. Section III describes the Hamiltonian of the charge transfer donor-acceptor complex which is employed as a case study in the present work, and Sec. IV details the quantum dynamical results. Finally, Sec. V summarizes and concludes. Several appendices add information on the derivation of the variational equations (Appendix A), the demonstration that energy conservation is not guaranteed with the present approach (Appendix B), and preliminary results for an adaptive propagation scheme that significantly reduces the number of trajectories (Appendix C).

\section{MULTI-CONFIGURATIONAL EHRENFEST APPROACH}

\section{A. Quantum-classical limit of the G-MCTDH method}

In Ref. 29, the G-MCTDH method, ${ }^{30,42,43}$ i.e., a Gaussianbased variant of $\mathrm{MCTDH},{ }^{27,28}$ is taken as a starting point to construct the quantum-classical limit of a GWP based multiconfigurational expansion. In the following, we briefly review the formulation of Ref. 29 and specify it to the case of nonadiabatic dynamics on coupled potential energy surfaces (PESs).

\section{General quantum-classical ansatz}

In Ref. 29, the following multiconfigurational G-MCTDH type wavefunction is considered:

$$
\Psi(\boldsymbol{r}, \boldsymbol{x}, t)=\sum_{j=1}^{J} \sum_{l=1}^{L} B_{j l}(t) \varphi_{j}(\boldsymbol{r}, t) g_{l}\left(\boldsymbol{x} ; \boldsymbol{\Lambda}_{l}(t)\right),
$$

where $\varphi_{j}(\boldsymbol{r}, t)$ represent single-particle functions $(\mathrm{SPFs})^{28}$ in the quantum subspace, labelled with the index $j=1, \ldots, J$, while $g_{l}\left(\boldsymbol{x} ; \boldsymbol{\Lambda}_{l}(t)\right)$ represent multidimensional Frozen Gaussian (FG) wavepackets, labelled with the index $l=1, \ldots, L$,

$$
\begin{aligned}
g_{l}\left(\boldsymbol{x} ; \boldsymbol{\Lambda}_{l}(t)\right)= & \exp \left[\left(\boldsymbol{x}-\boldsymbol{q}_{l}(t)\right)^{T} \boldsymbol{a}_{l}\left(\boldsymbol{x}-\boldsymbol{q}_{l}(t)\right)\right. \\
& \left.+i \boldsymbol{p}_{l}^{T}(t)\left(\boldsymbol{x}-\boldsymbol{q}_{l}(t)\right)+\mu_{l}(t)\right],
\end{aligned}
$$

where $\boldsymbol{x}=\left\{x_{1}, \ldots, x_{f}\right\}$ for an $f$-dimensional GWP particle and the time-dependent parameter vector $\boldsymbol{\Lambda}_{l}=\left\{\boldsymbol{q}_{l}, \boldsymbol{p}_{l}\right.$, $\left.\mu_{l}\right\}$ subsumes the GWP positions and momenta along with the complex phase $\mu_{l}$. According to the standard convention, the real part of $\mu_{l}$ is fixed by the normalization of the Gaussian wavepackets. ${ }^{30,42,43}$ In the FG ansatz, the width matrix $\boldsymbol{a}_{l}$ is a negative definite symmetric matrix, which in the present formulation is taken to be constant and diagonal, with $\left(\boldsymbol{a}_{l}\right)_{i i}<0$.

The wavefunction equation (1) represents a simple example of more complex multiconfigurational wavefunctions as described in Refs. 30, 42, and 43. Application of the DiracFrenkel Variational Principle (DFVP) ${ }^{44,45}$ to the coefficients $B_{j l}(t)$, quantum SPFs $\varphi_{j}(\boldsymbol{r}, t)$, and Gaussian parameters $\boldsymbol{\Lambda}_{l}(t)$ yields a set of coupled nonlinear equations entailing a nonclassical evolution of the Gaussian parameters $\boldsymbol{\Lambda}_{l}{ }^{30}$

In the quantum-classical limit, the FG wavepacket of Eq. (2) is effectively contracted to a phase-space point by a scaling procedure detailed in Ref. 29. To this end, a small parameter $\epsilon$ is introduced such that the $\epsilon \rightarrow 0$ limit defines the classical limit, ${ }^{29,46,47}$ noting that we formally use $\hbar=1 .{ }^{29}$ As a result, Eq. (1) yields a multiconfigurational quantum-classical wavefunction

$$
\Psi^{\epsilon}(\boldsymbol{r}, \boldsymbol{x}, t)=\sum_{j=1}^{J} \sum_{l=1}^{L} B_{j l}(t) \varphi_{j}(\boldsymbol{r}, t) e^{\frac{i}{\epsilon} S_{l}^{c l}(t)} g_{l}^{\epsilon}\left(\boldsymbol{x} ; \boldsymbol{q}_{l}(t), \boldsymbol{p}_{l}(t)\right)
$$

with a phase factor containing the classical action $S_{l}^{c l}(t)$ and the semiclassically scaled FG wavepacket

$$
\begin{aligned}
g_{l}^{\epsilon}\left(\boldsymbol{x} ; \boldsymbol{q}_{l}(t), \boldsymbol{p}_{l}(t)\right)= & N_{\boldsymbol{\epsilon}} \exp \left[\left(\boldsymbol{x}-\boldsymbol{q}_{l}(t)\right)^{T} \boldsymbol{a}_{l}\left(\boldsymbol{x}-\boldsymbol{q}_{l}(t)\right) / \epsilon\right. \\
& \left.+\frac{i}{\boldsymbol{\epsilon}} \boldsymbol{p}_{l}^{T}(t)\left(\boldsymbol{x}-\boldsymbol{q}_{l}(t)\right)\right] .
\end{aligned}
$$

These semiclassically scaled coherent-state wavepackets possess standard deviations in position $\sigma_{x}$ and momentum $\sigma_{p}$ that both scale like $\sqrt{\epsilon}^{46,47}$ such that they are "almost orthogonal" as $\epsilon$ approaches zero. ${ }^{29}$

\section{Formulation for coupled potential surfaces}

In the following, we will specifically consider the simplest case of a quantum subspace comprising a discrete 
electronic basis of $N$ states $|n\rangle, n=1, \ldots, N$, for a Hamiltonian that is assumed to take a generic diabatic vibronic-coupling form $^{48}$

$$
\begin{aligned}
\hat{H}_{\text {diabatic }}(\hat{\boldsymbol{x}}) & =\hat{T}_{\boldsymbol{x}} \hat{1}+\sum_{n, n^{\prime}=1}^{N}\left(h_{n, n^{\prime}}+\hat{V}_{n, n^{\prime}}(\hat{\boldsymbol{x}})\right)|n\rangle\left\langle n^{\prime}\right| \\
& \equiv \hat{T}_{\boldsymbol{x}} \hat{\hat{1}}+\sum_{n, n^{\prime}=1}^{N} \hat{H}_{n, n^{\prime}}(\hat{\boldsymbol{x}})|n\rangle\left\langle n^{\prime}\right|
\end{aligned}
$$

with the kinetic energy $\hat{T}_{x}$, the electronic Hamiltonian terms $h_{n, n^{\prime}}$, and the vibronic potential-type terms $\hat{V}_{n, n^{\prime}}(\hat{\boldsymbol{x}})$. The semiclassically scaled wavefunction of Eq. (3) now takes the form

$$
\left|\Psi^{\epsilon}(\boldsymbol{x}, t)\right\rangle=\sum_{n}^{N} \sum_{l}^{L} B_{n l}(t) e^{\frac{i}{\epsilon} \epsilon_{l}^{c l}(t)} g_{l}^{\epsilon}\left(\boldsymbol{x} ; \boldsymbol{q}_{l}(t), \boldsymbol{p}_{l}(t)\right)|n\rangle .
$$

In the context of MCTDH, this type of wavefunction ansatz corresponds to the so-called single-set form ${ }^{28}$ for nonadiabatic dynamics. Equation (6) also corresponds to a semiclassically scaled variant of a variational Multiconfigurational Gaussian $(\mathrm{vMCG})^{39,41,49}$ wavefunction in the single-set form. According to Ref. 29, application of the DFVP yields equations where the $B_{j l}$ coefficients are found to be decoupled for different $l \neq l^{\prime}$, i.e., configurations associated with different trajectories $\left(\boldsymbol{q}_{l}, \boldsymbol{p}_{l}\right)$ evolve independently, ${ }^{29}$

$$
i \dot{\boldsymbol{B}}_{l}=\boldsymbol{H}\left(\boldsymbol{q}_{l}\right) \boldsymbol{B}_{l},
$$

where $\boldsymbol{B}_{l}=\left(B_{1 l}, B_{2 l}, \ldots, B_{N l}\right)^{T}$ and the Hamiltonian matrix with elements $H_{n, n^{\prime}}\left(\boldsymbol{x}=\boldsymbol{q}_{l}(t)\right)$ relates to the quantum subspace Hamiltonian augmented by the interaction potential evaluated at the classical position $\boldsymbol{q}_{l}$,

$$
H_{n, n^{\prime}}\left(\boldsymbol{q}_{l}(t)\right)=h_{n, n^{\prime}}+V_{n, n^{\prime}}\left(\boldsymbol{x}=\boldsymbol{q}_{l}(t)\right),
$$

using that $\left.\left\langle g_{l}^{\epsilon}\right| \hat{V}_{n, n^{\prime}}(\hat{\boldsymbol{x}})\right)\left|g_{l}^{\epsilon}\right\rangle \approx V_{n, n^{\prime}}\left(\boldsymbol{q}_{l}\right)$ due to the narrow GWP width in the $\epsilon \rightarrow 0$ limit. ${ }^{29}$

From the DFVP, one further obtains classical evolution equations for the semiclassically scaled Gaussians,

$$
\dot{\boldsymbol{q}}_{l}=\boldsymbol{p}_{l}, \quad \dot{\boldsymbol{p}}_{l}=-\nabla_{\boldsymbol{q}_{l}} \bar{V}_{l}\left(\boldsymbol{q}_{l}\right),
$$

where the mean-field potential is given as

$$
\bar{V}_{l}\left(\boldsymbol{q}_{l}\right)=\left[\sum_{n}\left|B_{n l}\right|^{2}\right]^{-1} \sum_{n} \sum_{n^{\prime}} B_{n l}^{*} B_{n^{\prime} l} V_{n, n^{\prime}}\left(\boldsymbol{x}=\boldsymbol{q}_{l}\right) .
$$

In the following, we will employ the normalization condition $\sum_{n}\left|B_{n l}\right|^{2}=1$.

The above equations, i.e., Eq. (7) and Eqs. (9) and (10), correspond to the standard Ehrenfest equations ${ }^{1,2}$ such that the wavefunction of Eq. (6) can be interpreted as a superposition of independently evolving Ehrenfest configurations $\left|\Phi_{l}^{\mathrm{qc}}(\boldsymbol{x}, t)\right\rangle$,

$$
\left|\Psi^{\epsilon}(\boldsymbol{x}, t)\right\rangle=\sum_{l=1}^{L}\left|\Phi_{l}^{\mathrm{qc}}(\boldsymbol{x}, t)\right\rangle,
$$

where the quantum-classical (qc) Ehrenfest configurations are given by

$$
\left|\Phi_{l}^{\mathrm{qc}}(\boldsymbol{x}, t)\right\rangle=\sum_{n=1}^{N} B_{n l}(t) e^{\frac{i}{\epsilon} S_{l}^{c l}(t)} g_{l}^{\epsilon}\left(\boldsymbol{x} ; \boldsymbol{q}_{l}(t), \boldsymbol{p}_{l}(t)\right)|n\rangle .
$$

In the quantum-classical wavefunction [Eq. (11)], phase information is kept by attaching the phase factor $\exp \left(\frac{i}{\epsilon} S_{l}^{\mathrm{cl}}(t)\right)$ to each trajectory. However, due to the vanishing overlap of the trajectory-like $g_{l}^{\epsilon}$ functions, decoherence is effectively induced in the quantum subsystem.

\section{B. Quantum-classical scheme with finite-width wavepackets}

In applications to molecular systems, the vibrational motions are not truly classical, but rather of semiclassical nature. Therefore, we return to the finite-width GWPs of Eq. (2) and combine these with the classical-limit dynamics of Eqs. (7)-(10). When doing so, it turns out, though, that the resulting dynamics is inconsistent since the normalization of the wavefunction is not conserved.

To impose norm conservation, we associate an additional set of coefficients $A_{l}$ with each Ehrenfest configuration and determine these coefficients from the DFVP. The modified wavefunction ansatz is given as follows:

$$
|\Psi(\boldsymbol{x}, t)\rangle=\sum_{l=1}^{L} A_{l}(t)\left|\Phi_{l}(\boldsymbol{x}, t)\right\rangle,
$$

where the Ehrenfest configurations (now omitting the "qc" superscript) are given as

$$
\left|\Phi_{l}(\boldsymbol{x}, t)\right\rangle=\sum_{n=1}^{N} B_{n l}(t) g_{l}\left(\boldsymbol{x} ; \mathbf{\Lambda}_{l}(t)\right)|n\rangle .
$$

Here, the GWPs are taken to follow the classical equations of motion [Eq. (9)] even though they are in practice finite-width wavepackets. All phase factors have now been absorbed into the wavefunction coefficients such that the phase depending on the classical action no longer appears explicitly.

The resulting equations of motion read as follows (see Appendix A for details):

$$
\begin{aligned}
i \boldsymbol{S} \dot{\boldsymbol{A}} & =(\tilde{\boldsymbol{H}}-i \tau) \boldsymbol{A}, \\
i \dot{\boldsymbol{B}}_{l} & =\boldsymbol{H}\left(\boldsymbol{q}_{l}\right) \boldsymbol{B}_{l}, \\
\dot{\boldsymbol{q}}_{l} & =\boldsymbol{p}_{l}, \quad \dot{\boldsymbol{p}}_{l}=-\nabla_{\boldsymbol{q}_{l}} \bar{V}_{l}\left(\boldsymbol{q}_{l}\right),
\end{aligned}
$$

where Eqs. (16) and (17) are unchanged as compared with Eqs. (7) and (9), while the new equation [Eq. (15)] determines the variational time evolution of the $\boldsymbol{A}$ coefficients.

The above wavefunction ansatz, with variational coefficients $\boldsymbol{A}_{l}$ but pre-determined Ehrenfest configurations $\Phi_{l}$, is equivalent to the MCEv2 ansatz described by Shalashilin, ${ }^{26}$ except for the phase convention that is employed (see Appendix A). At this level of treatment, the method is not a genuine mixed quantum-classical propagation scheme, but rather an approximate quantum method.

All matrix elements appearing in Eq. (15) for the $\boldsymbol{A}$ coefficients are formulated in the basis of Ehrenfest configurations $\left|\Phi_{l}\right\rangle$ and read explicitly as follows: 


$$
\tilde{H}_{l k}=\left\langle\Phi_{l}|\hat{H}| \Phi_{k}\right\rangle=\sum_{n} \sum_{n^{\prime}} B_{n l}^{*} B_{n^{\prime} k}\left\langle n\left|\left\langle g_{l}|\hat{H}| g_{k}\right\rangle\right| n^{\prime}\right\rangle
$$

and similarly for the overlap matrix elements,

$$
S_{l k}=\left\langle\Phi_{l} \mid \Phi_{k}\right\rangle=\sum_{n} B_{n l}^{*} B_{n k}\left\langle g_{l} \mid g_{k}\right\rangle
$$

and for the differential overlap matrix elements,

$$
\tau_{l k}=\left\langle\Phi_{l} \mid \dot{\Phi}_{k}\right\rangle=\sum_{n}\left(B_{n l}^{*} \dot{B}_{n k}\left\langle g_{l} \mid g_{k}\right\rangle+B_{n l}^{*} B_{n k}\left\langle g_{l} \mid \dot{g}_{k}\right\rangle\right) .
$$

In the last two equations, we used the orthogonality of the primitive electronic basis $|n\rangle$. In Eq. (20), the derivative matrix element on the r.h.s. is evaluated using $\dot{g}_{k}=\sum_{\alpha}\left(\partial g_{k} / \partial \lambda_{k \alpha}\right) \dot{\lambda}_{k \alpha}$, where the parameters $\lambda_{k \alpha}$ constitute the parameter vector, $\boldsymbol{\Lambda}_{k}=\left\{\lambda_{k \alpha}\right\}$. As a result, all matrix elements can be expressed as Gaussian moments.

In contrast to Eqs. (7)-(9) which are variational in the quantum-classical limit $\epsilon \rightarrow 0$, Eqs. (15)-(17) are variational in a weaker sense. Specifically, Eq. (15) for the $\boldsymbol{A}$ coefficients is fully variational, but Eqs. (16) and (17) for the $\boldsymbol{B}$ coefficients and phase space parameters are pre-determined and variational in a weak sense, as demonstrated in Appendix A. There, it is shown that the application of the DFVP to a single Ehrenfest configuration yields Eqs. (16) and (17) if the phase relation $\operatorname{Im} \dot{\mu}=\boldsymbol{p} \cdot \dot{\boldsymbol{q}}$ is fulfilled. As already mentioned, the MCEv2 ansatz by Shalashilin ${ }^{26}$ employs a different phase convention (see Appendix A).

Another aspect that should be emphasized relates to the fact that Eqs. (15)-(17) conserve the wavefunction norm, but the energy expectation value is not a strictly conserved quantity in the MCE dynamics. This shortcoming is common to all methods that are based on classically moving Gaussians, ${ }^{50}$ in contrast to fully variational schemes like G-MCTDH or vMCG. As discussed in Appendix B, the energy drift should disappear in the limit where the Ehrenfest configurations form a complete basis set. However, this limit is hardly reached in practical applications. Even in our fully converged calculations, we observe an increase in energy of $\sim 100 \mathrm{meV}$ over a propagation time of $100 \mathrm{fs}$.

\section{APPLICATION TO DONOR-ACCEPTOR CHARGE TRANSFER SYSTEM}

In the following, the MCE method is applied to a donoracceptor (DA) system composed of an oligothiophene (OT 4 ) donor moiety and a fullerene $\left(\mathrm{C}_{60}\right)$ acceptor moiety. This system is a minimal model for the charge transfer in a paradigm DA system of organic photovoltaics, composed of poly-3hexylthiophene (P3HT) and phenyl- $\mathrm{C}_{61}$ butyric acid methyl ester (PCBM) components. ${ }^{51-56}$ The P3HT-PCBM system exhibits an ultrafast charge transfer, on a time scale of $\sim 50$ fs to $200 \mathrm{fs} .{ }^{52,53,57,58}$ In a previous study, ${ }^{31,59}$ we employed a Linear Vibronic Coupling (LVC) Hamiltonian in conjunction with the MCTDH method to describe the ultrafast, coherent electron transfer dynamics in this system. The Hamiltonian has been parameterized by electronic structure calculations using long-range-corrected density functional theory and the diabatization procedure described in Ref. 59, along with an ab initio generated spectral density of the phonon modes of the system.
The results presented here are obtained considering the data and procedures presented in Refs. 31 and 59.

\section{A. Hamiltonian and parametrization}

The abovementioned LVC model describes the coupling of an excitonic donor state $\left(\mathrm{OT}_{4}^{*}-\mathrm{C}_{60}\right)$, denoted $\mathrm{XT}$, to a charge separated state $\left(\mathrm{OT}_{4}^{+}-\mathrm{C}_{60}^{-}\right)$, denoted CT. This twostate Hamiltonian is a function of $N$ nuclear coordinates and can be cast in the form of a system-bath Hamiltonian,

$$
\hat{H}=\hat{H}_{0}+\hat{H}_{R}+\hat{H}_{B}
$$

where $\hat{H}_{0}$ refers to the electronic part, $\hat{H}_{R}$ is the part depending on the inter-fragment distance coordinate $R$, and $\hat{H}_{B}$ represents the collection of the $N_{\text {bath }}=N-1$ intra-molecular "bath" modes. ${ }^{31}$ The individual parts are given as

$$
\begin{gathered}
\hat{H}_{0}=-\Delta_{\mathrm{XT}-\mathrm{CT}}|\mathrm{CT}\rangle\langle\mathrm{CT}|+\gamma(|\mathrm{XT}\rangle\langle\mathrm{CT}|+| \mathrm{CT}\rangle\langle\mathrm{XT}|), \\
\hat{H}_{R}=\frac{\omega_{R}}{2}\left(\hat{R}^{2}+\hat{P}^{2}\right)+\kappa_{R} \hat{R}|\mathrm{CT}\rangle\langle\mathrm{CT}| \\
+\gamma_{R} \hat{R}(|\mathrm{XT}\rangle\langle\mathrm{CT}|+| \mathrm{CT}\rangle\langle\mathrm{XT}|), \\
\hat{H}_{B}=\sum_{i=1}^{N_{\text {bath }}} \frac{\omega_{i}}{2}\left(\hat{x}_{i}^{2}+\hat{p}_{i}^{2}\right)+\sum_{i=1}^{N_{\text {bath }}} \kappa_{i} \hat{x}_{i}|\mathrm{CT}\rangle\langle\mathrm{CT}|,
\end{gathered}
$$

where $\Delta_{\mathrm{XT}-\mathrm{CT}}$ is the electronic offset, $\gamma$ is the coordinateindependent part of the diabatic coupling, $\kappa_{R}$ and $\gamma_{R}$ are electronically diagonal and off-diagonal vibronic couplings due to the intermolecular $R$-mode, and $\left\{\kappa_{i}\right\}$ describe electronically diagonal vibronic couplings of the bath modes. The Hamiltonian equation (21) uses mass- and frequency-weighted coordinates. The diagonal vibronic coupling parameters $\kappa_{R}$ and $\left\{\kappa_{i}\right\}$ express the displacement of the CT equilibrium geometry from the XT reference geometry. The bath Hamiltonian $\hat{H}_{B}$ [Eq. (24)] represents the intra-molecular modes of the oligothiophene and fullerene fragments, which are diagonally coupled to the CT state. In line with Refs. 31 and 59, the reorganization energy term was not included in the model Hamiltonian.

The parameters appearing in $\hat{H}_{0}$ and $\hat{H}_{R}$ are given in Table I. The electron-phonon couplings $\left\{\kappa_{i}\right\}$ were determined by projecting the displacement between the XT and the CT minima onto the normal mode coordinates for the separate $\mathrm{OT}_{4}^{+}$and $\mathrm{C}_{60}^{-}$fragments. The normal mode analysis yields 246 normal modes in total for both fragments. ${ }^{31}$

As detailed in Ref. 31, the discrete distribution of the electron-phonon couplings has been used to construct a smooth spectral density $J(\omega)$ function, representing a continuous density of modes that gives a better description of the highdimensional polymer system. Here, we refer to the spectral density obtained with a Lorentzian broadening with a width parameter $\Delta=0.25 \Delta_{0}$, where $\Delta_{0}=4.4 \times 10^{-4}$ a.u. $\left(96 \mathrm{~cm}^{-1}\right)$

TABLE I. Parameters, given in eV, for the $\hat{H}_{\text {bath }}$ [Eq. (22)] and $\hat{H}_{R}$ [Eq. (23)] parts of the Hamiltonian $\hat{H}$ of Eq. (21).

\begin{tabular}{lcccc}
\hline \hline$\Delta_{\text {XT-CT }}$ & $\gamma$ & $\gamma_{\mathrm{R}}$ & $\kappa_{\mathrm{R}}$ & $\omega_{\mathrm{R}}$ \\
\hline 0.079 & 0.130 & -0.10 & 0.030 & 0.010 \\
\hline \hline
\end{tabular}




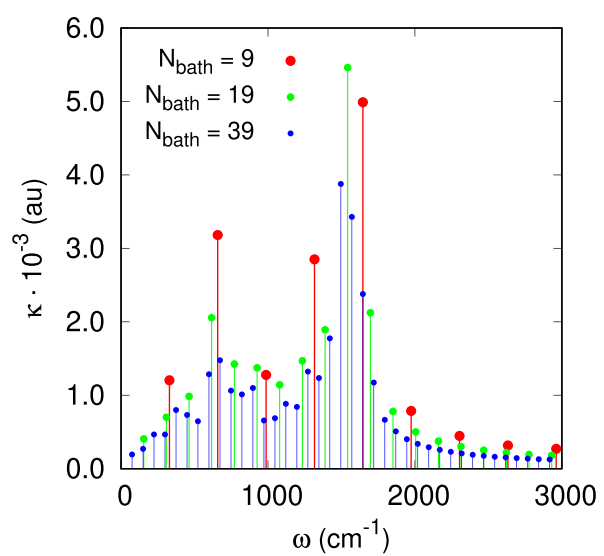

FIG. 1. Vibronic couplings $\left\{\kappa_{i}\right\}$ corresponding to different realizations of the discretized spectral density as discussed in the text, with $N_{\text {bath }}=9,19$, and 39 bath modes.

corresponds to the RMS of the sampling distance of the original data (see Ref. 31 for details). The continuous spectral density can then be re-discretized with an arbitrary number of $N_{\text {bath }}$ bath modes for an equidistant sampling interval $\Delta \omega$ such that several setups with variable dimensionality can be generated, ${ }^{60,61}$

$$
J(\omega)=\frac{\pi}{2} \sum_{i=1}^{N_{\text {bath }}} \kappa_{i}^{2} \delta\left(\omega-\omega_{i}\right),
$$

where the vibronic couplings depend on the sampling interval $\Delta \omega, \kappa_{i}=\left((2 / \pi) J\left(\omega_{i}\right) \Delta \omega\right)^{1 / 2}$.

In this work, three realizations of the Hamiltonian were obtained with $N_{\text {bath }}=9,19$, and 39 bath modes, shown in Fig. 1. The corresponding Poincaré recurrence times $\tau_{p}$ $=2 \pi / \Delta \omega$ are given as $101 \mathrm{fs}, 216 \mathrm{fs}$, and $446 \mathrm{fs}$. Therefore, in the first hundred femtoseconds of the dynamics, the different discretizations of the bath will give analogous converged benchmark results. The different bath sizes allow us to explore the effect of the dimensionality on the performance and the convergence of MCE.

\section{B. Potential energy curves}

In Fig. 2, the diabatic and adiabatic potential energy surfaces (PESs) pertaining to the LVC Hamiltonian are plotted as a function of the inter-fragment coordinate $R$ and an effective "Brownian oscillator" mode defined as $\hat{Q}=(1 / D) \sum_{i} \kappa_{i} \hat{x}_{i}{ }^{62}$ which subsumes the coupling of the bath modes to the electronic subsystem and determines the short-time dynamics. In our dynamical simulations, the wavepacket starts at the minimum of the XT potential, representing a relaxed exciton state. Due to the fact that the XT-CT coupling and the electronic offset take similar values, $\gamma \simeq \Delta_{\mathrm{XT}-\mathrm{CT}}$, the wavepacket starts to oscillate between the two diabatic states. Eventually, the system relaxes to a quasi-stationary state which is predominantly of CT character ( $~ 90 \%)$ with a non-negligible XT admixture $(\sim 10 \%)$. When evolving towards this asymptotic state, about $0.5 \mathrm{eV}$ of excess energy is transferred to the bath modes. At the same time, the $R$ coordinate is displaced to negative values, indicating a reduction of the inter-fragment distance. As illustrated by the time-evolving state-specific expectation values in Fig. 2, concerted oscillations of the XT and CT portions of the wavepacket are observed throughout the simulation interval in the diabatic picture of panel (a). [By contrast, the adiabatic dynamics of panel (b) shows a rapid depopulation of the upper adiabatic state, with weak oscillations of the residual population.]

\section{MCE PROPAGATION RESULTS}

In the following, we analyze MCE propagation results for several bath realizations as defined above, i.e., for $N=10$, $N=20$, and $N=40$ modes, where $N=N_{\text {bath }}+1$, including the $R$ mode. In Fig. 3, the MCE results are compared with the MCTDH benchmark results as well as statistical Ehrenfest results.

MCTDH reference calculations were performed in a similar fashion as described in Ref. 31 for a 60-mode realization of the system. A multi-set setup ${ }^{28}$ was used, with state-specific
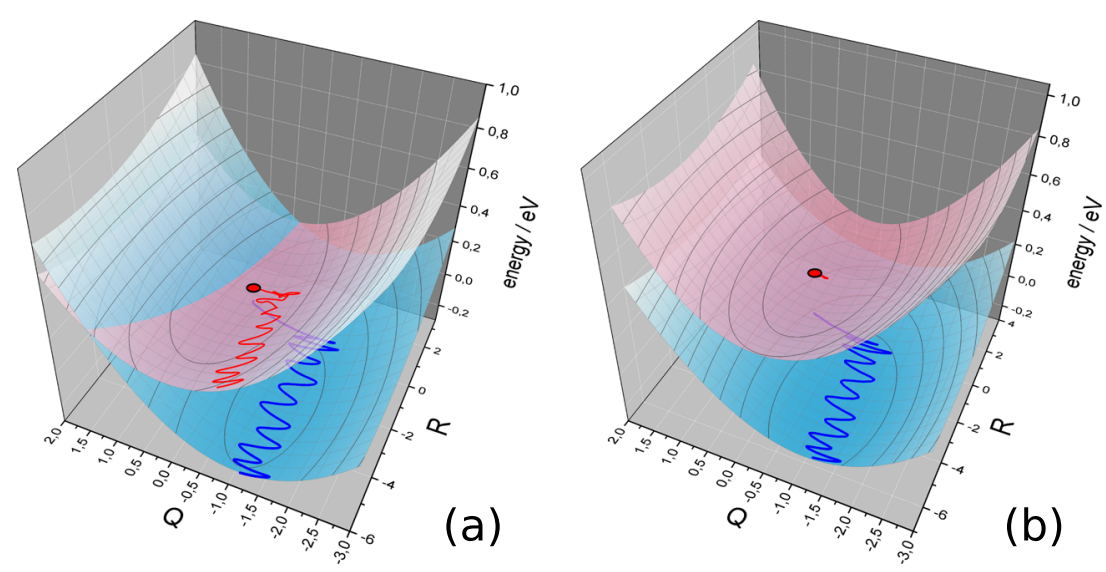

FIG. 2. Diabatic (a) and adiabatic (b) representations of the PESs pertaining to the Hamiltonian Eqs. (21)-(24) shown as a function of the $R$ coordinate and of an effective mode defined as $\hat{Q}=1 / D \sum_{i} \kappa_{i} \hat{x}_{i}{ }^{62}$ which subsumes the coupling of the bath modes to the electronic subsystem. The trajectories correspond to state-specific expectation values in the diabatic vs. adiabatic representation, departing from the initial condition on the XT surface, marked by a circle. The concerted oscillatory motion in the diabatic representation [panel (a)] indicates that the wavepacket exhibits a sustained coherent superposition of the portions belonging to the two diabatic states. By contrast, the residual wavepacket portion in the upper adiabatic state essentially remains stationary [see panel (b)]. Adiabatic coordinate expectation values were calculated for a 20 -mode system using a simplified diabatic-to-adiabatic transformation along the paths defined by the full set of time-evolving coordinate expectation values. 

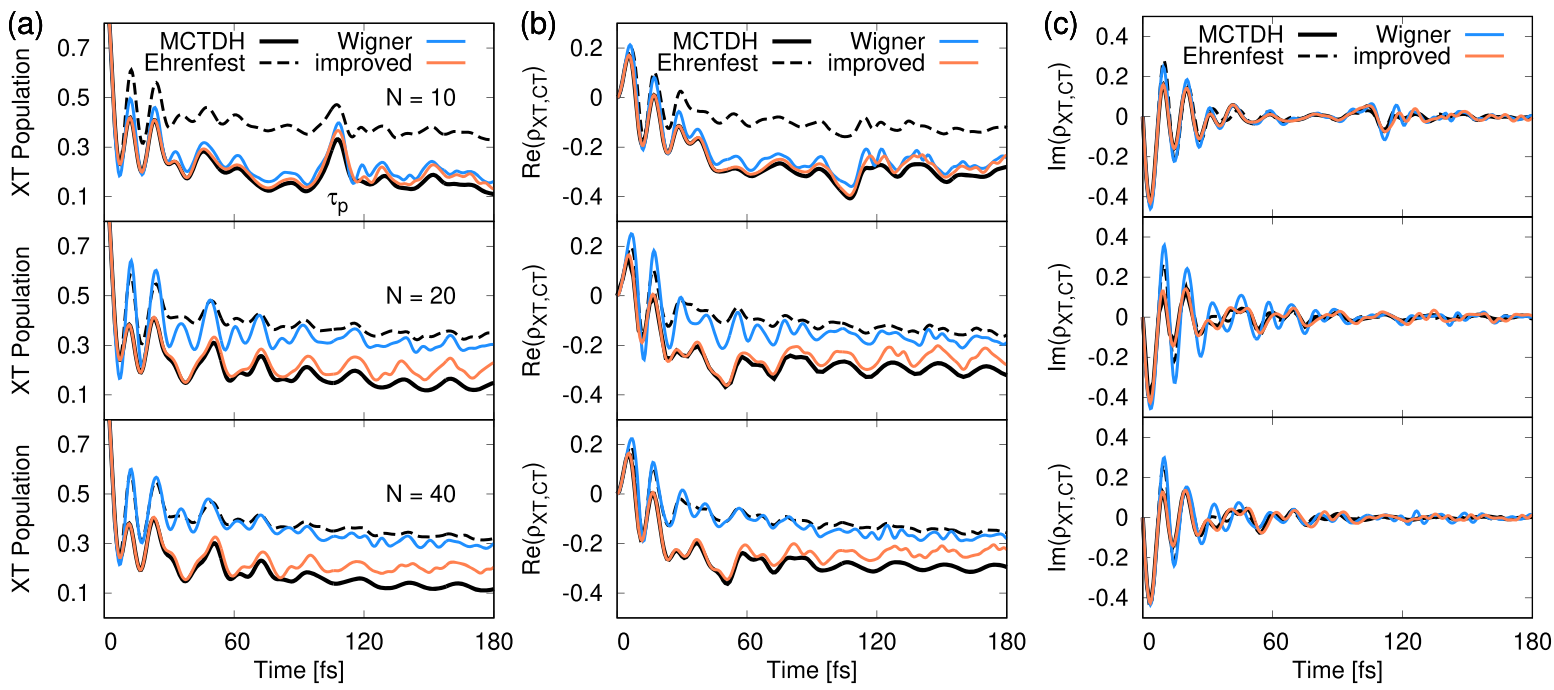

FIG. 3. Time-dependent XT state population (a) and real and imaginary parts of the electronic coherence $\rho_{\mathrm{XT}, \mathrm{CT}}$ [(b) and (c), respectively], are plotted for different sizes of the bath $(N=10,20$, and 40, panels from top to bottom). Results have been obtained with MCTDH (solid black line), statistical Ehrenfest (dashed black line) and MCE with both standard Wigner and improved sampling (blue and orange lines, respectively). Only the best MCE results are shown, with 5001 trajectories for the standard sampling and 2001 for the improved scheme. In the upper panel of (a), $\tau_{P}$ denotes the Poincare recurrence time in the $N=10$ case.

SPF sets. The intermolecular $R$ mode was represented by a one-dimensional subspace with 6 to 8 SPFs, while mode combination was used for the set of bath modes. Typically, 5-dimensional subspaces (combined particles) were employed for the bath modes, with 3 to 9 SPFs per particle.

In the following discussion, we compare time-evolving state populations as well as electronic coherences which play a key role in the dynamics,

$$
\rho_{\mathrm{XT}, \mathrm{CT}}(t)=\operatorname{Tr}\{|\mathrm{CT}\rangle\langle\mathrm{XT}| \hat{\rho}(t)\} .
$$

Here, the imaginary part $\operatorname{Im} \rho_{\mathrm{XT}, \mathrm{CT}}$ determines the transient state-to-state population flux, $\Gamma_{\mathrm{XT}, \mathrm{CT}}=-2 \gamma \operatorname{Im} \rho_{\mathrm{XT}, \mathrm{CT}}$ where $\gamma$ is the diabatic coupling, while the real part $\operatorname{Re} \rho_{\text {XT,CT }}$ captures the quasi-stationary XT-CT superposition that was mentioned above. $^{31}$

In the following, we discuss general features of the observed dynamics, before turning to the details of the MCE results.

\section{A. General features of the dynamics-MCTDH and Ehrenfest results}

Figure 3 shows the time-evolving XT population [panel (a)], the real part of the electronic coherence [panel (b)], and the imaginary part of the electronic coherence [panel (c)], for MCE and statistical Ehrenfest calculations, along with $\mathrm{MCTDH}$ reference results. As can be seen from the MCTDH results, the initial oscillatory decay of the XT state [panel (a)] mirrors the time-evolving profile of the imaginary part of the electronic coherence, $\operatorname{Im} \rho_{\mathrm{XT}, \mathrm{CT}}$, which decays to zero within about 50 fs [panel (c)]. Beyond this time, the XT state population correlates with the real part of the electronic coherence, $\operatorname{Re} \rho_{\text {XT,CT }}$ [panel (b)], which tends towards a quasi-stationary value indicating that the lower adiabatic state is reached within approximately $100 \mathrm{fs}$. Apart from residual oscillations which result from the finite size of the bath, the XT population converges to an asymptotic value of $\sim 0.1$ that coincides with the XT character of the lower adiabatic state obtained by diagonalization (cf. Fig. 2). For $N=10$, a revival peak is present at $\sim 100 \mathrm{fs}$, consistent with the Poincaré recurrence time of $\tau_{p}=101$ fs for the 9-mode discretization of the bath. Apart from this, we see that the MCTDH results for $N=20$ and $N=40$ are very similar, indicating that the dynamics is converged with respect to the bath size.

Figure 3 also shows the results of standard statistical Ehrenfest calculations, obtained as averages over $L=5000$ realizations, using Wigner function sampling. In this case, too, the XT population can be considered converged with respect to the bath size. However, the comparison with the quantum benchmark is far from satisfactory. Interestingly, the imaginary part of the electronic coherence $\operatorname{Im} \rho_{\mathrm{XT}, \mathrm{CT}}(\mathrm{t})$ [panel (c)] is described quite accurately, but the initial transient XT population decay appears displaced and the asymptotic value of the XT population lies around $\sim 0.4$, much larger than the correct quasi-equilibrium value. In line with this, the real part of the coherence, $\operatorname{Re} \rho_{\mathrm{XT}, \mathrm{CT}}$, is significantly displaced [see panel (b)]. As mentioned in the Introduction, this reflects a wellknown shortcoming of Ehrenfest dynamics, i.e., the violation of the principle of detailed balance ${ }^{19,20}$ and failure to correctly describe the XT vs. CT participation in the asymptotic state. A key issue is therefore the question whether the MCE method is able to repair this misrepresentation of the final superposition state.

\section{B. MCE propagation}

For the MCE propagation, initial conditions were constructed such that a single Gaussian wavepacket is initially occupied, as in the MCTDH reference calculations. Two different sampling schemes were considered: First, standard Wigner sampling, where the initial configurations and momenta of the Gaussian centers are determined by sampling the Wigner 
(a)

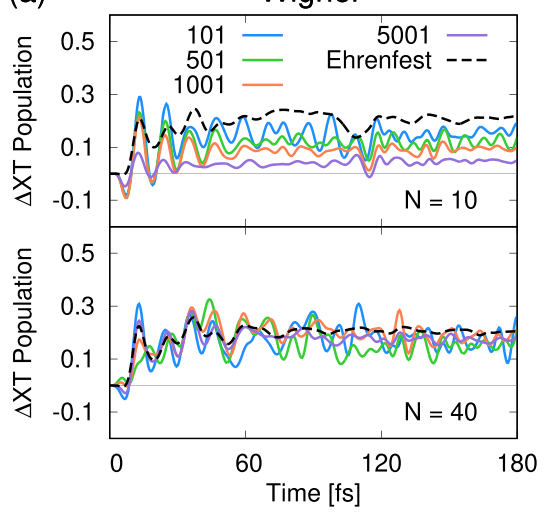

(b)

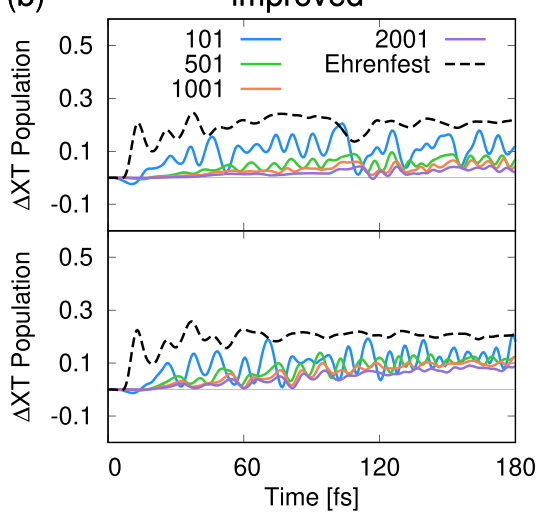

FIG. 4. Convergence of the XT populations computed with MCE as compared with MCTDH benchmark results. Differences from the benchmark are shown for the standard Wigner sampling [panel (a)] and for the improved scheme [panel (b)], respectively. Top and bottom panels refer to different sizes of the bath ( $N=10$ and 40, respectively) and curves are color-coded for the different numbers of trajectories used. Convergence is clearly superior for the improved sampling scheme. distribution associated with the initial wavefunction, as in the statistical Ehrenfest simulations. Second, sampling with a compression factor, as detailed below, similar to the scheme suggested by Shalashilin and collaborators in the context of the Coupled Coherent States (CCS) method $^{63}$ as well as MCE. ${ }^{25,33}$ We also use a random sampling of the electronic amplitudes $\boldsymbol{B}_{l}$, as suggested in Ref. 33. Details of the compression scheme are described in Sec. IV C. These two sampling schemes will be labeled "Wigner sampling" vs. "improved sampling" in the following.

Figure 3 reports our best MCE results, together with the MCTDH reference results and Ehrenfest calculations. The MCE results were obtained with a set of $L=5001$ trajectories for the Wigner sampling and $L=2001$ trajectories for the improved sampling.

For $N=10$, MCE with both Wigner sampling and improved sampling is in qualitatively good agreement with the reference $\mathrm{MCTDH}$ results, very much in contrast to the Ehrenfest calculations. MCE with improved sampling tends to perform best and is in excellent agreement with the MCTDH benchmark during the first 100 fs. Beyond 100 fs, convergence seems to become more difficult.

In the $N=20$ and $N=40$ systems, a significant difference between the Wigner sampling and improved sampling conditions becomes apparent. Indeed, improved sampling is found to give results that are close to the MCTDH benchmark, whereas the Wigner sampling results rather approach the Ehrenfest case. In contrast to the $N=10$ case, it seems that decoherence dominates if Wigner sampling is employed. As in the Ehrenfest calculations, the imaginary part of the coherence is essentially correctly reproduced in all cases, while the real part-describing the asymptotic coherent superposition state-is flawed when Wigner sampling is employed.

Complementary to Fig. 3, Fig. 4 demonstrates the convergence of the XT population as a function of the number of trajectories, for Wigner sampling [panel (a)] vs. improved sampling [panel (b)]. The difference with respect to the reference MCTDH results is shown.

Focusing on the Wigner sampling case [panel (a)], we obtain a uniform, although rather slow, convergence for $N=10$ (upper panel): by increasing the number of configurations, the MCE results approaches the MCTDH results, spurious oscillatory features are reduced, and the asymptotic limit tends to the exact equilibrium value. As the number of dimensions increases to $N=40$ (lower panel), however, we practically see no convergence. With a larger number of Ehrenfest configurations, some oscillations are washed out, but the curves for $L=5001$ differ only slightly from the statistical Ehrenfest results.

For the improved sampling case [panel (b)], convergence is clearly more favorable. Here, the compression factors that have been applied vary depending on the bath dimensionality, as listed in Table II. Specifically, in all cases, we have adopted the maximum value attainable without incurring numerical problems due to near-singularities of the overlap matrix. The optimal compression had to be increased for larger systems to counteract the effect of the decay of overlap with the number of dimensions.

The results of Figs. 3 and 4 show that the improved sampling scheme gives a remarkable improvement within the first $100 \mathrm{fs}$ of the dynamics. The comparison with the MCTDH benchmark shows that the first oscillations of the XT population are captured even with a few hundred configurations. We stress again that with the standard Wigner sampling, we were not able to reach a similar level of accuracy, not even with $L=5000$ trajectories.

At longer times, though, i.e., beyond $\sim 100 \mathrm{fs}$, even the improved MCE results start to deviate from the MCTDH benchmark, as mentioned above. This reflects how challenging it is for MCE to represent a coherent superposition of wavepackets evolving on near-parallel PESs. Since the computational cost rises significantly as a function of $L$, it is difficult to go beyond the present calculations (see Sec. IV D for details on the numerical effort of the present MCE implementation).

TABLE II. Optimal values of the compression parameter $\sigma$ adopted in the MCE calculations described in the text. The table also reports the corresponding values of the RMS overlap between 2000 Ehrenfest configurations, set by the improved sampling scheme described in the text.

\begin{tabular}{lrc}
\hline \hline$N$ & $\sigma$ & RMS overlap \\
\hline 10 & 4 & 0.551 \\
20 & 25 & 0.733 \\
40 & 100 & 0.744 \\
\hline \hline
\end{tabular}




\section{Interpretation in terms of Gaussian overlap}

The difficulty of convergence for larger $N$ and the sensitivity to the compression parameter can be understood by an analysis of the overlap between the GWP basis functions. As suggested by Ref. 64 , the quality of a Gaussian basis set can be measured in terms of a non-orthogonality per Gaussian, related to the Frobenius norm ${ }^{65}$ of the overlap matrix, that in the case of random initial conditions is conveniently defined as the root mean squared (RMS) overlap $\bar{O}_{\mathrm{RMS}}$,

$$
\bar{O}_{\mathrm{RMS}}^{2}=\frac{1}{2 N(N-1)} \sum_{i>j}\left|\left\langle g_{i} \mid g_{j}\right\rangle\right|^{2} .
$$

As we will show in the following, in our setup, the initial value of $\bar{O}_{\text {RMS }}$ converges to a well-defined limit by increasing the number of Gaussian functions.

The absolute value of the overlap between two GWP functions as defined in Eq. (2), centered in phase-space at $\left(\boldsymbol{q}_{1}, \boldsymbol{p}_{1}\right)$ and $\left(\boldsymbol{q}_{2}, \boldsymbol{p}_{2}\right)$ and where we use $\boldsymbol{a}_{l}=-\boldsymbol{\Omega}$, is given by

$$
\begin{aligned}
\left|\left\langle g_{1} \mid g_{2}\right\rangle\right|= & \exp \left(-\frac{1}{2}\left(\boldsymbol{q}_{2}-\boldsymbol{q}_{1}\right) \boldsymbol{\Omega}\left(\boldsymbol{q}_{2}-\boldsymbol{q}_{1}\right)\right. \\
& \left.-\frac{1}{8}\left(\boldsymbol{p}_{2}-\boldsymbol{p}_{1}\right) \boldsymbol{\Omega}^{-1}\left(\boldsymbol{p}_{2}-\boldsymbol{p}_{1}\right)\right)
\end{aligned}
$$

and thus depends only on the relative position $\left(\boldsymbol{q}_{1}-\boldsymbol{q}_{2}\right)$ and relative momentum $\left(\boldsymbol{p}_{1}-\boldsymbol{p}_{2}\right)$ of the two functions. For the MCE results described above, the initial conditions are defined by randomly sampling the phase-space centers according to a Wigner distribution, which in this case is a Gaussian distribution in both coordinates and momenta, with covariances given as $\boldsymbol{\Sigma}_{q}=\frac{1}{4} \boldsymbol{\Omega}^{-1}$ and $\boldsymbol{\Sigma}_{p}=\boldsymbol{\Omega}$, respectively. Given that the difference between two normally distributed random variables is also normally distributed, the variance is the sum of the component variances. This allows us to predict the distribution of $\left(\boldsymbol{q}_{1}-\boldsymbol{q}_{2}\right)$ and $\left(\boldsymbol{p}_{1}-\boldsymbol{p}_{2}\right)$ and to integrate Eq. (28) to obtain the RMS overlap as

$$
\bar{O}_{\text {RMS }}=2^{-N / 2} .
$$

This analysis shows that regardless of the number of initial configurations, the overlap decays exponentially with the dimension of the system: $\bar{O}_{\mathrm{RMS}}$ is $3.13 \cdot 10^{-2}$ for $N$ $=10,9.77 \cdot 10^{-4}$ for $N=20$, and $9.54 \cdot 10^{-7}$ for $N=40$. By increasing the dimension $N$, we converge towards a condition where both the overlap and the Hamiltonian matrix elements between any two Gaussian functions are negligible such that the configurations become effectively uncoupled in the equation for the $\boldsymbol{A}$ coefficients [cf. Eq. (15)]. Under these conditions, MCE becomes essentially equivalent to the statistical Ehrenfest approach.

To counteract the intrinsic loss of overlap-and, hence, coherence-between the basis functions, we introduce a scaling of the initial position and momentum distribution, in order to obtain neighboring Gaussians with an increased overlap, as suggested by Shalashilin. ${ }^{33,63}$ Specifically, we introduce a compression parameter $\sigma$ in the phase-space standard deviations of the initial sampling distribution, $\boldsymbol{\Sigma}_{q}=(1 / 4 \sigma) \boldsymbol{\Omega}^{-1}$ and $\boldsymbol{\Sigma}_{p}=(1 / \sigma) \boldsymbol{\Omega}$, such that the RMS overlap becomes

$$
\bar{O}_{\mathrm{RMS}}=\left(\sigma^{-2}+1\right)^{-N / 2} .
$$

By increasing the value of $\sigma$, we are now able to tune the initial overlap as desired. The effect of the compression $\sigma$ is analogous to what has been observed by Poirier and Light ${ }^{64}$ by optimizing the relative distance of a (nonstochastic) uniformly distributed set of Gaussian functions used as a static basis for the solution of the time-independent Schrödinger equation. The authors found that by decreasing the grid spacing, the accuracy of the solution improves. As we showed above, in our case, too, the optimal distribution of the Gaussian functions features highly overlapping basis sets.

We now consider the effect of the compression parameter on the RMS overlap in the course of the dynamics. In Fig. 5, the RMS values are shown as a function of time for the different bath models considered and for representative values of $\sigma$. We note that the overlap between the Ehrenfest configurations not only measures the phase-space distance of the Gaussians but also the dephasing of the electronic amplitudes. The plot highlights that the compression has a determining effect on the magnitude of the overlap, which goes from being negligible for small $\sigma$ to a significant value - of the order of $\sim 0.1-$ for the largest $\sigma$ that we could apply without incurring linear dependencies. Figure 5 also shows that there is a decrease of the overlap with time although on a scale which is much reduced as compared with the one set by different compression values. The approximately exponential decay is a consequence of the fact that the basis functions, guided by the Ehrenfest equations of motion, expand to a larger region of phase space in the nuclear coordinates. As a function of time, the moving basis set therefore becomes less suitable to represent the asymptotic superposition state.



FIG. 5. RMS overlap between the Ehrenfest configurations as a function of time, computed with MCE for different sizes of the bath $(N=10,20$, and 40 , from top to bottom) and different values of the compression parameter $\sigma$ (color coded as described in the legend of each panel). Results are plotted on a semi-log scale. 

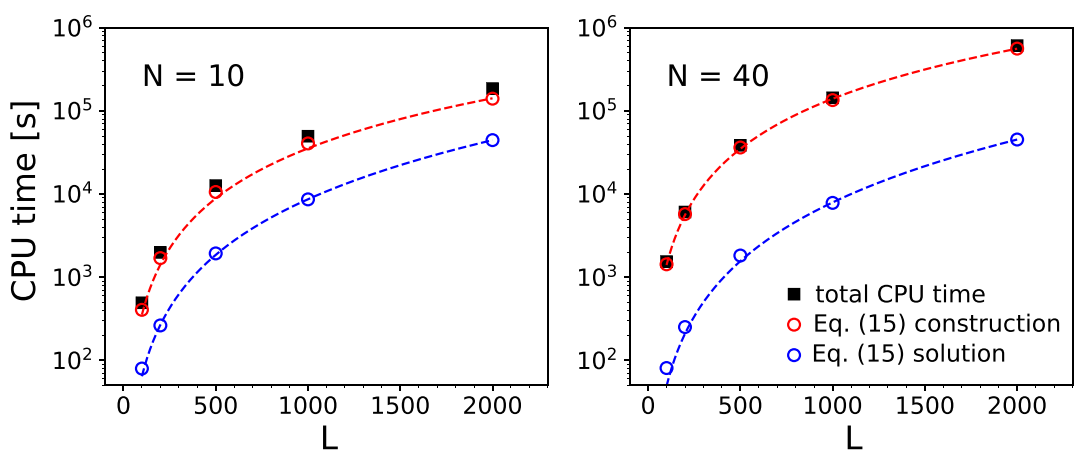

FIG. 6. CPU times of the MCE calculations carried out with the improved sampling scheme, as a function of the number of Ehrenfest configurations $L$, and for different sizes of the bath $(N=10,40)$; the CPU times refer to an Intel Xeon E5-2690 v4 node with 256 GB memory. Total CPU times (black squares) are reported along with the CPU time of the two most expensive parts of the algorithm, i.e., the construction of the overlap matrix $S$ and the r.h.s. of Eq. (15) (red dots) and the solution of Eq. (15) (blue dots). Red and blue dashed lines show the quadratic and cubic models fitting the recorded CPU times.

The comparison with the MCTDH benchmark results reported in Fig. 3 shows that MCE is capable of describing the coherent transients and concomitant population transfer very accurately. However, as already mentioned, a discrepancy in the real part of $\rho_{\text {XT,CT }}$ starts to appear beyond $\sim 100 \mathrm{fs}$, while the system converges towards a stationary state. When the two portions of the wavepacket are well separated on the two diabatic states, $\operatorname{Re} \rho_{\text {Xт,СT }}$ approaches an incorrect asymptotic value, with an error that is slightly lowered by the enlargement of the basis set. This situation appears to be particularly challenging for MCE since the correct representation of the electronic coherence requires that the basis functions on the two diabatic surfaces maintain a precise phase relationship, which is difficult to achieve with an incomplete, moving basis set. This problem is emphasized by the increase of the system size.

\section{Computational cost of MCE calculations}

In our implementation of MCE, the two computationally most expensive parts are (i) the calculation and storage of the terms appearing in Eq. (15) for the propagation of the $\boldsymbol{A}$ vector and (ii) the solution of this equation as a system of linear equations to obtain the derivative vector $\dot{\boldsymbol{A}}$. These two steps of the computation have different scaling properties: the former has a leading term that is a quadratic function $\sim L^{2}$ of the number of configurations, whereas the latter follows a cubic scaling $\sim L^{3}$. Figure 6 reports the central processing unit (CPU) times of these two steps, determined for the calculations with the improved sampling scheme.

For the values of $N$ and $L$ considered in the MCE calculations, it turns out that the first of the above steps, i.e., the construction of Eq. (15), is the most expensive part, even though the second step, in principle, exhibits the dominant $\sim L^{3}$ scaling properties. This must be due to the large prefactor of the first step, which again comes as a surprise, since all the terms involved can be expressed as a function of 1D Gaussian moments, which are computed analytically. In fact, it is the extremely large number of these terms that is the reason for the computational effort, particularly for high dimensional systems with a large basis of Ehrenfest configurations.
The resulting computational cost, as illustrated in Fig. 6, clearly limits the applicability of MCE to higher-dimensional systems. For comparison, CPU times for the MCTDH reference calculations were far more advantageous. Specifically, the overall number of MCTDH configurations and CPU times, for a 200 fs propagation interval, was $n_{\text {config }}=7000$ (CPU time: $725 \mathrm{~s}$ ) for the 10 -mode system, $n_{\text {config }}=162528$ (CPU time: $18540 \mathrm{~s}$ ) for the 20-mode system, and $n_{\text {config }}=1905408$ (CPU time: $12631 \mathrm{~s}$ ) for the 40-mode system.

In an attempt to alleviate this problem, we designed and tested algorithms to reduce the computational cost, e.g., by skipping the computation of matrix elements that are expected to be negligible based on the phase-space distance between the Gaussian centers. These attempts, however, were not conclusive so far because few matrix elements are in fact negligible when a compression factor is applied to the Wigner distribution of the initial Gaussians.

As an alternative route to reducing the number of configurations, we further carried out preliminary investigations of an adaptive MCE propagation scheme, as reported in Appendix C. This direction appears particularly promising to us, even though the present implementation is not yet efficient.

\section{CONCLUSIONS}

In the present paper, we employed a multiconfiguration Ehrenfest type method which can be derived from the classical limit of the Gaussian-based MCTDH method, following Ref. 29. When taking into account finite-width GWP functions, one naturally obtains variational equations which are closely related to the so-called MCEv2 approach by Shalashilin, ${ }^{26}$ except for a time-evolving phase factor. This approach employs superpositions of independently evolving Ehrenfest configurations, in contrast to the MCEv1 approach ${ }^{25}$ where these configurations are permitted to interact. While the latter approach is more flexible, MCEv2 is very convenient to use due to the independent evolution of the configurations. Hence, MCEv 2 has been employed in on-the-fly AIMC-MCE calculations $\mathrm{s}^{32}$ and was also found to perform well in an earlier study of nonadiabatic dynamics in pyrazine ${ }^{26}$ as well as a recent investigation of a spin-boson system, ${ }^{33}$ provided the sampling is suitably adapted. Here, we focused on an MCEv2 
type method in view of its connection to the classical-limit description of Ref. 29.

The results that we have presented show that MCE is in general able to faithfully represent the nonadiabatic dynamics under study, in contrast to the standard Ehrenfest approximation. The latter correctly describes the earliest transients but fails to capture the superposition of diabatic states that prevails at later times, reflecting the failure of the Ehrenfest approach to describe detailed balance. MCE is found to perform well as long as the phase-space density of the Gaussian centers is maintained high enough to obtain a good representation of the wavefunction. The RMS overlap of Ehrenfest configurations provides a suitable measure for this density. For the $N=10$ mode system investigated here, the configurational overlap remains large enough if a standard Wigner sampling is employed. For higher-dimensional realizations of the system-notably $N=20$ and $N=40$ - an enhanced sampling of the initially unoccupied Gaussian functions was necessary by using a compression parameter in line with Refs. 25, 26, 32, and 33. For these larger systems, it remains challenging to represent coherences at longer times, as the Gaussian wavepackets are driven apart by the classical dynamics on the different diabatic states. In the cases shown here, between $L=2000$ and $L=5000$ Ehrenfest configurations were employed - a rather large number as compared with variational GWP approaches, but at the same time a small number as compared with semiclassical initial value representation (IVR) methods. 66

Given that a statistical analysis shows that the overlap between the basis functions decays exponentially with the dimensionality of the system, a much larger number of configurations would be necessary in systems with hundreds of modes. In practice, this is unfeasible due to the computational cost of MCE (see Sec. IV D), which requires solving the variational equations for the $\boldsymbol{A}$ coefficients in the basis of $L$ configurations, involving a scaling up to $L^{3}$, to compute the inverse of the overlap matrix. This suggests that the method should be generalized to a multi-layer approach, similar to the multilayer Gaussian MCTDH (ML-GMCTDH) scheme. ${ }^{67}$ Indeed, recent two-layer (2L)-GMCTDH calculations for the donoracceptor system studied in the present work have been shown to yield very good results for system sizes up to 100 modes, at moderate computational cost. ${ }^{68}$

Based on these considerations, we can draw the following conclusions. First, MCE can be used to obtain a fully quantum description that systematically improves upon the statistical Ehrenfest approach. The case study considered here is challenging in this respect since a coherent superposition of diabatic states persists on longer time scales, resulting in a clear-cut failure of the Ehrenfest approach.

Second, the present study underscores the crucial role of the initial sampling for systems exceeding a minimal dimensionality, as already shown by Shalashilin and collaborators. ${ }^{32,33}$ These authors also propose additional, more advanced sampling techniques like "cloning" and "bit-bybit" propagation. ${ }^{33}$ Given that the present system exhibits a rather regular classical dynamics, the compression approach was shown to work in a satisfactory fashion. It remains open, though, to what extent enhanced sampling techniques would lead to an accurate dynamics in strongly anharmonic situations.

From the viewpoint of fully variational GWP methods like the G-MCTDH and vMCG approaches, the sensitivity of MCE to the initial sampling is a crucial drawback. Indeed, the former methods are largely insensitive to the distribution of initially unoccupied basis functions since the variational evolution is able to "optimize" the basis set as a function of time. Conversely, fully variational methods are more demanding in the implementation and more susceptible to linear dependencies. Suitable hybrid methods might be designed by which a fully variational quantum core wavefunction is combined with an MCE-type description of a larger number of less important modes.

\section{ACKNOWLEDGMENTS}

We thank Rainer Hegger for useful discussions and Robert Binder and Praveen Budakoti for help in preparing the figures. The German-Israeli Foundation for Scientific Research and Development is acknowledged for support of this project under Grant No. GIF I-1337-302.5/2016. M.B. gratefully acknowledges fellowship support by the Alexander von Humboldt Foundation.

\section{APPENDIX A: DERIVATION OF MCE EQUATIONS OF MOTION}

Here, we present a derivation of the MCE equations for finite-width GWPs, Eqs. (15)-(17). With regard to the wavefunction ansatz [Eq. (13)], the variation of the wavefunction $|\delta \Psi\rangle$ is restricted to the $A_{l}$ coefficients, $|\delta \Psi\rangle=\sum_{l} \delta A_{l}\left|\Phi_{l}\right\rangle$, given that the Ehrenfest configurations $\left|\Phi_{l}(\boldsymbol{x}, t)\right\rangle$ are pre-determined as further discussed below. This yields for each individual and independent variation $\delta A_{l}$,

$$
\begin{aligned}
& \delta A_{l}^{*}\left\langle\Phi_{l}\left|\hat{H}-i \frac{\partial}{\partial t}\right| \Psi\right\rangle=0 \\
& \delta A_{l}^{*} \sum_{k}\left[A_{k}\left\langle\Phi_{l}|\hat{H}| \Phi_{k}\right\rangle-i \dot{A}_{k}\left\langle\Phi_{l} \mid \Phi_{k}\right\rangle-i A_{k}\left\langle\Phi_{l} \mid \dot{\Phi}_{k}\right\rangle\right]=0
\end{aligned}
$$

Noting that the Ehrenfest configurations are not orthogonal, we introduce the overlap matrix, $S_{l k}=\left\langle\Phi_{l} \mid \Phi_{k}\right\rangle$, and the differential overlap $\tau_{l k}=\left\langle\Phi_{l} \mid \dot{\Phi}_{k}\right\rangle$, which plays the role of the gauge in the wavefunction ansatz. Hence, Eq. (A1) yields

$$
\begin{aligned}
i \sum_{k} S_{l k} \dot{A}_{k} & =\sum_{k}\left(\left\langle\Phi_{l}|\hat{H}| \Phi_{k}\right\rangle-i \tau_{l k}\right) A_{k} \\
& =\sum_{k}\left(\tilde{H}_{l k}-i \tau_{l k}\right) A_{k},
\end{aligned}
$$

which corresponds to Eq. (15) of the main text. These types of coefficient equations generally arise in the case of a non-orthogonal basis, for example, in the context of AIMS, G-MCTDH, and vMCG; see also the discussion of Ref. 50.

In contrast to the equation of motion (EOM) for the $\boldsymbol{A}$ coefficients, Eq. (16) for the $\boldsymbol{B}$ coefficients is not variational in a strict sense. However, this equation can be understood as a 
variational equation in a weak sense, by referring to a singleconfigurational formulation. Notably, omitting the summation over configurations $l$ in Eq. (13), we consider a single Ehrenfest configuration

$$
|\Psi(x, t)\rangle=|\Phi(x, t)\rangle=\sum_{n} B_{n} g(x ; \Lambda(t))|n\rangle .
$$

The variational equations of motion for the configuration $|\Psi(\boldsymbol{x}, t)\rangle$ are again derived from the DFVP, $\langle\delta \Phi|i d / d t-\hat{H}| \Phi\rangle$ $=0$, where $|\delta \Phi\rangle=\sum_{n} \delta B_{n} g(\boldsymbol{x} ; \Lambda(t))|n\rangle+\delta g \sum_{n} B_{n}|n\rangle$ is an arbitrary variation of the wavefunction ansatz of Eq. (A3). When projecting along the variation of the $m$ th electronic amplitude $B_{m}$, we obtain

$$
i \dot{B}_{m}=\sum_{n}\left\langle g\left|H_{m n}\right| g\right\rangle B_{n}-i\langle g \mid \dot{g}\rangle B_{m} .
$$

With a classical approximation for the nuclear motion, i.e., assuming that $g(\boldsymbol{x})$ has a negligible extension in coordinate space, the Hamiltonian can be evaluated at the center of the Gaussian $\left\langle g\left|H_{m n}\right| g\right\rangle \approx H_{m n}(\boldsymbol{q})$ and the last formula can be identified with the MCE equation of motion of the $\boldsymbol{B}$ coefficients, Eq. (16).

The last term of Eq. (A4) depends on the differential overlap of the Gaussian function, $\langle g \mid \dot{g}\rangle$, which is computed as follows from the GWP ansatz Eq. (2), and using that $\operatorname{Re}(\mu)$ is defined by the normalization:

$$
\langle g \mid \dot{g}\rangle=i(-\boldsymbol{p} \cdot \dot{\boldsymbol{q}}+\operatorname{Im} \dot{\mu}) .
$$

This equation highlights that the definition of the complex phase-factor $\mu$ in Eq. (2) is conceptually equivalent to the choice of the orbital gauge in MCTDH. ${ }^{28}$ Any imaginary part $\operatorname{Im}(\mu)$ will leave the normalization of the GWP function unaffected,

$$
\frac{d}{d t}\langle g \mid g\rangle=\langle g \mid \dot{g}\rangle+\langle g \mid \dot{g}\rangle^{*}=0 .
$$

However, different choices of $\operatorname{Im}(\mu)$ will give rise to different dynamics of the Gaussians, as made evident by Eq. (A4). Specifically, we define $\operatorname{Im}(\mu)$ by the equation of motion

$$
\operatorname{Im} \dot{\mu}=\boldsymbol{p} \cdot \dot{\boldsymbol{q}} .
$$

This leads to $\langle g \mid \dot{g}\rangle=0$ such that Eq. (A4) coincides with Eq. (16), $i \dot{B}_{m}=\sum_{n} H_{m n}(\boldsymbol{q}) B_{n}$.

By contrast, an alternative phase definition has been adopted in Ref. $25, \operatorname{Im} \mu=\boldsymbol{p} \cdot \boldsymbol{q} / 2$ such that Eq. (A4) becomes

$$
i \dot{B}_{m}=\sum_{n} H_{m n}(\boldsymbol{q}) B_{n}+\frac{1}{2}(\boldsymbol{q} \cdot \dot{\boldsymbol{p}}-\boldsymbol{p} \cdot \dot{\boldsymbol{q}}) B_{m} .
$$

From a numerical viewpoint, both formulations Eq. (16) and Eq. (A8) perform similarly.

In the final step, Eq. (A4) yields the classical EOMs [Eq. (17)] for the GWP parameters when the variation $\delta g$ for a single GWP as defined by the ansatz Eq. (A3) is considered.

\section{APPENDIX B: ENERGY CONSERVATION FOR TIME-DEPENDENT BASIS SETS}

While a fully variational dynamics, as in the G-MCTDH and vMCG methods, preserves the wavefunction norm and energy ${ }^{28}$ this is not the case for the weakly variational EOMs Eqs. (15)-(17). These EOMs are norm-preserving but only approximately conserve energy, as detailed in the following (see also Ref. 50).

The energy expectation value of the MCE wavefunction is given as $E=\langle\Psi|H| \Psi\rangle$, with the wavefunction of Eqs. (13) and (14). The time derivative of this expectation value corresponds to the energy drift at each time of the dynamics, i.e.,

$$
\frac{d E}{d t}=2 \operatorname{Re}(\langle\Psi|H| \dot{\Psi}\rangle)
$$

leading to the explicit expression ${ }^{50}$

$$
\frac{d E}{d t}=2 \operatorname{Re}\left(\sum_{l l^{\prime}} A_{l}^{*}\left\langle\Phi_{l}|H| \dot{\Phi}_{l^{\prime}}\right\rangle A_{l^{\prime}}+A_{l}^{*}\left\langle\Phi_{l}|H| \Phi_{l^{\prime}}\right\rangle \dot{A}_{l^{\prime}}\right)
$$

The time-derivative of the $A_{l}$ coefficients is given by Eq. (15) such that $d E / d t$ can be further rewritten as

$$
\begin{aligned}
\frac{d E}{d t}= & 2 \operatorname{Re}\left(\sum_{l l^{\prime}} A_{l}^{*}\left\langle\Phi_{l}|H| \dot{\Phi}_{l^{\prime}}\right\rangle A_{l^{\prime}}+\frac{1}{i} \boldsymbol{A}^{\dagger} \tilde{\boldsymbol{H}} \boldsymbol{S}^{-1} \tilde{\boldsymbol{H}} \boldsymbol{A}\right. \\
& \left.-\sum_{l l^{\prime}} \sum_{k k^{\prime}} A_{l}^{*}\left\langle\Phi_{l}|H| \Phi_{k}\right\rangle\left(\boldsymbol{S}^{-1}\right)_{k k^{\prime}}\left\langle\Phi_{k^{\prime}} \mid \dot{\Phi}_{l^{\prime}}\right\rangle A_{l^{\prime}}\right) .
\end{aligned}
$$

In the last equation, the second term on the r.h.s. is purely imaginary, since

$$
\left(\frac{1}{i} \boldsymbol{A}^{\dagger} \tilde{\boldsymbol{H}} \boldsymbol{S}^{-1} \tilde{\boldsymbol{H}} \boldsymbol{A}\right)^{*}=-\frac{1}{i} \boldsymbol{A}^{\dagger} \tilde{\boldsymbol{H}} \boldsymbol{S}^{-1} \tilde{\boldsymbol{H}} \boldsymbol{A},
$$

and thus does not contribute to the real part of Eq. (B3). Furthermore, we recognize that the last term contains a projector on the non-orthogonal Ehrenfest configurations,

$$
P=\sum_{k k^{\prime}}\left|\Phi_{k}\right\rangle\left(\boldsymbol{S}^{-1}\right)_{k k^{\prime}}\left\langle\Phi_{k^{\prime}}\right|
$$

In light of the above, we rewrite Eq. (B3) as

$$
\frac{d E}{d t}=2 \operatorname{Re}\left(\sum_{l l^{\prime}} A_{l}^{*}\left\langle\Phi_{l}|H(1-P)| \dot{\Phi}_{l^{\prime}}\right\rangle A_{l^{\prime}}\right) .
$$

The last formula indicates that the energy drift is determined by the matrix elements $\left\langle\Phi_{l}|H(1-P)| \dot{\Phi}_{l^{\prime}}\right\rangle$, which are trivially zero when the Ehrenfest configurations form a complete set. In fact, under this assumption, we can consider $P=1$ and the r.h.s. of Eq. (B6), vanishes accordingly. In general, however, the derivative of the functions $\Phi_{l^{\prime}}$ will not be entirely spanned by the other Ehrenfest configurations and the matrix elements of Eq. (B6) will differ from zero, which in turn implies that the expectation value of the energy will change over time.

In the derivation of Eq. (B6), we did not make use so far of the actual EOM of the Ehrenfest configuration. As a consequence, the above discussion applies to any type of ansatz with a time-dependent basis set. However, a fully variational dynamics guarantees energy conservation because in this case the EOM can be cast in the general form

$$
i \sum_{l} \dot{\Phi}_{l} A_{l}=(1-P) H \sum_{l} \Phi_{l} A_{l}
$$


where the projector ensures that the variational EOM of the functions $\Phi_{l}$ captures the first order propagation of the wavefunction, excluding the part that is already described by the variation of the linear coefficients $A_{l}$. Substituting the last formula into Eq. (B6), we obtain a symmetric purely imaginary term, thereby making the energy drift vanish.

\section{APPENDIX C: ADAPTIVE SCHEME BASED ON FIRST-ORDER VARIATION}

The discussion of Appendix B highlights that MCE is necessarily less accurate than a fully variational propagation method. As a systematic approach to improve upon MCE, we explored an adaptive algorithm where unoccupied Ehrenfest configurations are added during the dynamics, in the attempt to enforce a wavefunction evolution that remains as close as possible to the first-order variation in time.

The idea that a higher-order time-variation of the wavefunction can be used to optimize the choice of unoccupied configurations has been recently proposed by Manthe ${ }^{69}$ in view of improving the initial MCTDH propagation. However, in the present context, there are two main differences. First, MCTDH is variational and exact to first order such that the estimate of the error is based on the difference with the second-order variation. In our case, the first variation is not exactly represented by the MCE propagation such that a correction at the level of the first order is sought for. Second, in Ref. 69, a second-order error estimate is used in the context of a method with a fixed number of configurations, to optimize the initial conditions for the unoccupied basis functions. In our case, we will use the first-order error estimate in the context of an adaptive scheme, to guide the addition of new configurations at any step of the dynamics.

Specifically, the exact first-order variation of a wavefunction $\psi$ after a time $\delta t$ is

$$
\delta \psi^{\text {exact }}=\frac{1}{i} H \psi \delta t,
$$

whereas the variation corresponding to the MCE equations of motion is given by

$$
\delta \psi^{\mathrm{MCE}}=\left(\frac{1}{i} P H \psi+(1-P) \sum_{l} \dot{\Phi}_{l} A_{l}\right) \delta t,
$$

where the last formula was obtained similarly to the formula for the energy drift [cf. Eq. (B3)] by substituting the MCE equations of motion into the derivative of the wavefunction. The difference between these two variations is then proportional to the vector

$$
\begin{aligned}
\frac{i}{\delta t} \Delta(\delta \psi) & =\frac{i}{\delta t}\left(\delta \psi^{\text {exact }}-\delta \psi^{\mathrm{MCE}}\right) \\
& =(1-P)\left[H \psi-\sum_{l}\left(i \dot{\Phi}_{l}\right) A_{l}\right] \\
& =(1-P)\left[\sum_{l}\left(H \Phi_{l}-i \dot{\Phi}_{l}\right) A_{l}\right] .
\end{aligned}
$$

The last formula shows that the inclusion of unoccupied configurations (i.e., configurations with $A_{l}=0$ ) does not contribute to the summation in the square parentheses and therefore cannot directly increase the first-order accuracy. However, additional configurations will enlarge the space spanned by the basis functions and consequently reduce the range of the complementary projector $(1-P)$, diminishing the error.

In our adaptive scheme, we adopt a measure of the firstorder error estimate that corresponds to the squared norm of the vector of Eq. (C3),

$$
\begin{aligned}
\frac{1}{\delta t^{2}}\|\Delta(\delta \psi)\|^{2}= & \sum_{l l^{\prime}} A_{l}^{*}\left\langle H \Phi_{l}-i \dot{\Phi}_{l} \mid H \Phi_{l^{\prime}}-i \dot{\Phi}_{l^{\prime}}\right\rangle A_{l^{\prime}} \\
& -\sum_{l l^{\prime}} A_{l}^{*}\left\langle H \Phi_{l}-i \dot{\Phi}_{l}|P| H \Phi_{l^{\prime}}-i \dot{\Phi}_{l^{\prime}}\right\rangle A_{l^{\prime}}
\end{aligned}
$$

Since the addition of unoccupied configurations does not modify the first term of the sum, we can equivalently minimize the norm of the error by maximizing the second term

$$
\begin{aligned}
\xi & =\sum_{l l^{\prime}} A_{l}^{*}\left\langle H \Phi_{l}-i \dot{\Phi}_{l}|P| H \Phi_{l^{\prime}}-i \dot{\Phi}_{l^{\prime}}\right\rangle A_{l^{\prime}} \\
& =\boldsymbol{A}^{\dagger}(\tilde{\boldsymbol{H}}-i \boldsymbol{\tau})^{\dagger} \boldsymbol{S}^{-1}(\tilde{\boldsymbol{H}}-i \boldsymbol{\tau}) \boldsymbol{A},
\end{aligned}
$$

which is a combination of the matrices employed for the propagation of the $\boldsymbol{A}$ vectors.

Using the error estimate outlined above, we implemented an adaptive scheme in which new configurations are generated at each propagation step, with a phase space center that is close to the ones of the occupied configurations and with randomly chosen electronic amplitudes. The configuration that gives the largest reduction of the first-order error estimate is selected and numerically optimized to give the maximum value of $\xi$. If this exceeds a given threshold, the trial configuration is added to the actual wavefunction.

In Fig. 7, the results obtained with this strategy are compared with the results computed with the improved sampling described in the main text, for the 20-dimensional model potential. In the adaptive scheme, we start with a single Ehrenfest configuration with $A_{1}=1$ and configurations are added until 1000 trajectories are reached. Comparison with the best results obtained for 1000 trajectories using the "improved" scheme shows that a similar accuracy is achieved, with a slightly better description of the long time asymptotic value of the XT population.

The results presented here prove that the first-order error estimate can be effectively used to guide an adaptive



FIG. 7. XT state population plotted as a function of time for the model with $N=20$ modes. Results were obtained with MCTDH (solid black line) and with $1000 \mathrm{MCE}$ trajectories using two different strategies, i.e., the improved sampling described in the main text (light vermillion line) and the adaptive scheme described in Appendix C (violet line). 
implementation of MCE, even if the algorithm that we described above has limited practical use. One of the main advantages of an adaptive scheme is that the short time dynamics is described with a much smaller number of configurations, hence with a significant saving of computation time (e.g., in the calculations reported in Fig. 7, the integration required $20.34 \mathrm{~h}$ for the standard sampling vs. $11.45 \mathrm{~h}$ for the adaptive scheme). However, in the present implementation, this CPU time reduction is overcompensated by the cost of the algorithm that is used to determine the positions of the new configurations $(14.39 \mathrm{~h})$. Therefore, work is currently in progress to find an optimal and efficient scheme to construct and select configurations that minimize the first-order error estimate.

${ }^{1}$ J. B. Delos, W. R. Thorson, and S. K. Knudson, Phys. Rev. A 6, 709 (1972). ${ }^{2}$ G. Billing, Chem. Phys. Lett. 100, 535 (1983).

${ }^{3}$ J. L. Alonso, A. Castro, J. Clemente-Gallardo, J. C. Cuchi, P. Echenique, and F. Falceto, J. Phys. A: Math. Theor. 44, 395004 (2011).

${ }^{4}$ J. L. Alonso, J. Clemente-Gallardo, J. C. Cuchi, P. Echenique, and F. Falceto, J. Chem. Phys. 137, 054106 (2012).

${ }^{5}$ J. C. Tully and R. K. Preston, J. Comput. Phys. 55, 562 (1971).

${ }^{6}$ J. C. Tully, J. Chem. Phys. 93, 1061 (1990).

${ }^{7}$ S. Hammes-Schiffer and J. C. Tully, J. Comput. Phys. 101, 4657 (1994).

${ }^{8}$ J. C. Tully, Faraday Discuss. 110, 407 (1998).

${ }^{9}$ M. Head-Gordon and J. C. Tully, J. Chem. Phys. 103, 10137 (1995).

${ }^{10}$ C. C. Martens and J.-Y. Fang, J. Chem. Phys. 106, 4918 (1997).

${ }^{11}$ R. Kapral and G. Ciccotti, J. Comput. Phys. 110, 8919 (1999).

12 A. Nassimi, S. Bonella, and R. Kapral, J. Comput. Phys. 133, 134115 (2010).

${ }^{13}$ S. Bonella, G. Ciccotti, and R. Kapral, Chem. Phys. Lett. 484, 399 (2010).

${ }^{14}$ F. Agostini, S. Caprara, and G. Ciccotti, Europhys. Lett. 78, 30001 (2007).

${ }^{15}$ J. Caro and L. Salcedo, Phys. Rev. A 60, 842 (1999).

${ }^{16}$ P. A. M. Dirac, Math. Proc. Cambridge Philos. Soc. 26, 376 (1930).

${ }^{17}$ R. B. Gerber, V. Buch, and M. A. Ratner, J. Chem. Phys. 77, 3022 (1982),

${ }^{18}$ R. B. Gerber and M. A. Ratner, J. Phys. Chem. 92, 3252 (1988).

${ }^{19}$ P. V. Parandekar and J. C. Tully, J. Chem. Theory Comput. 2, 229 (2006)

${ }^{20}$ A. Jain and J. E. Subotnik, J. Phys. Chem. A 122, 16 (2018).

${ }^{21}$ P. V. Parandekar and J. C. Tully, J. Chem. Phys. 122, 094102 (2005).

${ }^{22}$ J. R. Schmidt, P. V. Parandekar, and J. C. Tully, J. Chem. Phys. 129, 044104 (2008).

${ }^{23}$ C. C. Martens, J. Phys. Chem. Lett. 7, 2610 (2016).

${ }^{24}$ L. Wang, A. E. Sifain, and O. V. Prezhdo, J. Phys. Chem. Lett. 6, 3827 (2015).

${ }^{25}$ D. V. Shalashilin, J. Chem. Phys. 130, 244101 (2009).

${ }^{26}$ D. V. Shalashilin, J. Chem. Phys. 132, 244111 (2010).

${ }^{27}$ H. Meyer, U. Manthe, and L. S. Cederbaum, Chem. Phys. Lett. 165, 73 (1990).

${ }^{28}$ M. H. Beck, A. Jäckle, G. A. Worth, and H. Meyer, Phys. Rep. 324, 1 (2000).

${ }^{29}$ S. Römer and I. Burghardt, Mol. Phys. 111, 3618 (2013).

${ }^{30}$ I. Burghardt, H.-D. Meyer, and L. S. Cederbaum, J. Chem. Phys. 111, 2927 (1999).

${ }^{31}$ H. Tamura, R. Martinazzo, M. Ruckenbauer, and I. Burghardt, J. Chem. Phys. 137, 22A540 (2012).

${ }^{32}$ D. V. Makhov, C. Symonds, S. Fernandez-Alberti, and D. V. Shalashilin, Chem. Phys. 493, 200 (2017).

${ }^{33}$ C. Symonds, J. A. Kattirtzi, and D. V. Shalashilin, J. Chem. Phys. 148, 184113 (2018).
${ }^{34}$ T. J. Martínez, M. Ben-Nun, and R. D. Levine, J. Phys. Chem. 100, 7884 (1996).

${ }^{35}$ M. Ben-Nun and T. J. Martínez, J. Phys. Chem. A 103, 10517 (1999).

${ }^{36}$ M. Ben-Nun and T. J. Martínez, Adv. Chem. Phys. 121, 439 (2002).

${ }^{37}$ A. M. Virshup, C. Punwong, T. V. Pogorelov, B. A. Lindquist, C. Ko, and T. J. Martínez, J. Phys. Chem. B 113, 3280 (2009).

${ }^{38}$ G. A. Worth and I. Burghardt, Chem. Phys. Lett. 368, 502 (2003).

${ }^{39}$ G. A. Worth, M. Robb, and I. Burghardt, Faraday Discuss. 127, 307 (2004).

${ }^{40}$ D. Mendive-Tapia, B. Lasorne, G. A. Worth, M. A. Robb, and M. J. Bearpark, J. Chem. Phys. 137, 22A548 (2012).

${ }^{41}$ G. Richings, I. Polyak, K. Spinlove, G. Worth, I. Burghardt, and B. Lasorne, Int. Rev. Phys. Chem. 34, 269 (2015).

${ }^{42}$ I. Burghardt, M. Nest, and G. A. Worth, J. Chem. Phys. 119, 5364 (2003).

${ }^{43}$ I. Burghardt, K. Giri, and G. A. Worth, J. Chem. Phys. 129, 174104 (2008).

${ }^{44}$ C. Lubich, From Quantum to Classical Molecular Dynamics: Reduced Models and Numerical Analysis (European Mathematical Society Publishing House, Zuerich, Switzerland, 2008).

${ }^{45} \mathrm{M}$. Bonfanti and I. Burghardt, Chem. Phys. 515, 252 (2018).

${ }^{46}$ G. A. Hagedorn, Ann. Inst. H. Poincaré Phys. Théor. 42, 363 (1985).

${ }^{47}$ G. A. Hagedorn, Ann. Phys. 269, 77 (1998).

${ }^{48}$ H. Köppel, W. Domcke, and L. S. Cederbaum, Adv. Chem. Phys. 57, 59 (1984)

${ }^{49}$ M. Bonfanti, J. Petersen, P. Eisenbrandt, I. Burghardt, and E. Pollak, J. Chem. Theory Comput. 14, 5310 (2018).

${ }^{50}$ S. Habershon, J. Chem. Phys. 136, 014109 (2012).

${ }^{51}$ N. S. Sariciftci, L. Smilowitz, A. J. Heeger, and F. Wudl, Science 258, 1474 (1992).

${ }^{52}$ C. J. Brabec, G. Zerza, G. Cerullo, S. De Silvestri, S. Luzzati, J. C. Hummelen, and S. Sariciftci, Chem. Phys. Lett. 340, 232 (2001).

${ }^{53}$ S. De, T. Kesti, M. Maiti, F. Zhang, O. Inganäs, A. Yartsev, T. Pascher, and V. Sundström, Chem. Phys. 350, 14 (2008).

${ }^{54}$ P. Parkinson, J. Lloyd-Hughes, M. B. Johnston, and L. M. Herz, Phys. Rev. B 78, 115321 (2008).

${ }^{55}$ R. D. Pensack, K. M. Banyas, L. W. Barbour, M. Hegadorn, and J. B. Asbury, Phys. Chem. Chem. Phys. 11, 2575 (2009).

${ }^{56}$ R. D. Pensack and J. B. Asbury, J. Phys. Chem. Lett. 1, 2255 (2010).

${ }^{57}$ R. D. Pensack, K. M. Banyas, and J. B. Asbury, J. Phys. Chem. B 114, 12242 (2010).

${ }^{58}$ M. Tong, N. E. Coates, D. Moses, A. J. Heeger, S. Beaupré, and M. Leclerc, Phys. Rev. B 81, 125210 (2010).

${ }^{59}$ H. Tamura, I. Burghardt, and M. Tsukada, J. Phys. Chem. C 115, 10205 (2011).

${ }^{60}$ R. Martinazzo, K. H. Hughes, F. Martelli, and I. Burghardt, Chem. Phys. 377, 21 (2010).

${ }^{61}$ M. Bonfanti, K. H. Hughes, I. Burghardt, and R. Martinazzo, Ann. Phys. 527, 556 (2015).

${ }^{62}$ K. H. Hughes, C. Christ, and I. Burghardt, J. Chem. Phys. 131, 124108 (2009).

${ }^{63}$ D. V. Shalashilin and M. S. Child, J. Chem. Phys. 128, 054102 (2008).

${ }^{64}$ B. Poirier and J. C. Light, J. Chem. Phys. 113, 211 (2000).

${ }^{65}$ G. H. Golub and C. F. Van Loan, Matrix Computations, 3rd ed. (Johns Hopkins, Baltimore, MD, 1996).

${ }^{66}$ M. S. Child, Semiclassical Mechanics with Molecular Applications (Oxford University Press, Oxford, UK, 2014).

${ }^{67}$ S. Römer, M. Ruckenbauer, and I. Burghardt, J. Chem. Phys. 138, 064106 (2013).

${ }^{68}$ P. Eisenbrandt, M. Ruckenbauer, and I. Burghardt, J. Chem. Phys. 149, 174102 (2018).

${ }^{69}$ U. Manthe, J. Chem. Phys. 142, 244109 (2015). 